\title{
Study on Landscape Space Construction of Relics Park
}

\author{
Li Yan \\ Huanghe Science and Technology College \\ Zhengzhou, China
}

\begin{abstract}
As an important method of site protection, historic park is being paid more and more attention in protection and utilization of site. However, since the development of site protection is still in the early stage lack of theoretical guidance when there are many problems such as unobvious thematic feature, isolated site or inconformity with the local culture, weak landscape attraction. Based on the principle of park space planning, the contents and building methods of landscape of relics park have been studied in this paper at two aspects of culture landscape space and physical landscape space, in order to provide a new idea and method for landscape planning of relics park.
\end{abstract}

Keywords—relics park; culture landscape; physical landscape

\section{INTRODUCTION}

Relics park is a site protection mode based on relics protection which has a function to take landscape planning. Therefore, in order to make a good planning of relics park, the relics display and park landscape planning should be combined together based on relics protection so as to protect the site itself and site environment, furthermore through building culture connotation and historical site specific culture atmosphere in accordance with the relics, the culture values of sites are spread fully.

\section{LANDSCAPE PLANNING PRINCIPLES OF RELICS PARK}

According to dynamic development characteristics, spatial layout inherent characteristics, spatial functional characteristics, culture characteristics of relics parks, the principles of relics park space planning are summarized as follows:

\section{A. Respect for Site Development Process}

Since the site environment has been formed during a long term of history, we should take respect for the site body and carriers left during history in scope of the site, as well as surrounding environment which has important influence on the site characteristics.

\section{B. Respect for Original Spatial Frameworks of Sites}

Site internal space has its inherent spatial layout which is often composed of internal road link, each part of which is an activity place required at the time of building. Thus each site

Fund project: Research project training for young key teachers of Henan colleges in 2016: the protection and innovation of landscape design of relics park in Henan. plaque has its own functional characteristics that should be no free to change in site protection, which is shown by the authenticity of site that is one of the targets of park space planning.

\section{Respect for Landscape Environment Intention}

Sites are formed in a historical process of completion and evolution, and displayed through archaeological dig by contemporary people. Furthermore, site protection including site environmental background which represents natural environment, historical social and culture environment related with the site. Therefore, the space planning of relics park must follow the landscape environment intention, in order to express the original site landscape intention, show the original scene of the site, and protect the ecological environment of the site.

\section{Respect for Landscape Culture Patches of Site}

One of the biggest natures of the site is its culture value, which is the feature compared to other landscapes. The large site is composed of multiple landscape patches each of which has its own special meaning. All culture patches should be present based on the overall culture theme in order to show particularity of the relics park.

\section{CUlture LANDSCAPE SPACE PlanNing OF RElics PARK}

\section{A. Extract Culture Theme of Relics Park}

Since the most important display of site is not the site entities, but the deep structure expression of space which is namely spirit expressed by the entities, space planning should both express culture spirit in overall, but also express features and deep from tiny things, which requires that the overall culture characteristics of the site should be summarized, as well as the features of site culture plaque should be refined.

The first step of relics park planning is refined culture theme that determines the overall characteristics and culture, which should focus on the integrity and uniqueness of culture in that the concluded culture theme has infectious and influence. In order to do this, depth and connotation of the culture should be understood. Based on deeply understanding of the site culture, firstly refine the characteristics of the site and its culture plaques, finally determine its culture theme combined with the overall ambience and scale of the site, specify its planning concept. Therefore, the establishment 
process of culture theme is concluded of "research - refining express" in this paper.

\section{B. Culture Theme Expression of Relics Park}

For the expression of site culture theme, firstly understand the beauty of the site, in displaying its culture theme, often through expressing its artistic conception to build space theme.

1) Overall planning: The establishment of overall planning is the leader of a set of planning system. The overall planning of relics park is an overall concept and layout of the relics park development, is the culture theme over the site connotation, based on deeply researching site culture, regional development and other characteristics. The establishment of relics park overall planning has provided is a direction to refine partition concept.

2) Project planning concept: Because the site landscape space is composed of a plurality of culture plaques each of which mostly contains unique features or culture, the project planning concept of site is provided in view of culture plaques. In building project planning concept, firstly we should understand the cultural plaques are the parts of cultural theme, are the interpretation of culture theme from different aspects and levels, namely each project planning concept should has common feature; secondly, understand the uniqueness of each cultural plaque, so as to establish different cultural theme in different project planning, which means that the concept of each project is unique planning.

\section{3) Intentional expression techniques}

The ultimate embodiment of site culture is reflected through various means of expression. In general, the project express of core area of relics park project is the most appropriate cultural theme, because its planning and design are closely around site protection and display. The expression of surrounding environment requires a combination of ingenious design according to the cultural elements refined from site culture, to highlight different characteristics of different partitions at the same time, but also to make whole park culture unity by the same or similar expressions. In general, from planning to project planning to express intention, the site culture theme is indispensable, with different emphasis on each level, expressing artistic conception beauty of the site, and building the uniqueness of the relics park to improve its influence and appeal.

\section{RELICS PARK ENTITy LANDSCAPE SPACE PlanNING}

\section{A. Overall Layout}

Perceiving space environment has a feature of integrity both felling of forms and realizing of deeper contents. Space environment can affect people's psychological activities through effecting human's visual and behavior. Therefore, for physical landscape space planning of relics park, firstly we must grasp its macro level, and summarize its planning content and research methods as a whole. According to the three elements of "plaque, corridor, matrix" of landscape space, the overall space layout of relics park can be divided into the point layout, strip layout and surface layout.
The point layout aims at the site plaques that have relatively independences which is the core of relics park landscape and has strong cohesion and clear landscape structure level.

Strip layout is based on the site has a long narrow and more concentrated site landscape corridor, has a strong functional continuity in function, thereby forming a unified whole.

The surface layout site has large space, rich spatial levels and complex spatial layout, which is conducive to the unified planning and protection of relics park.

\section{B. Functional Site Space Layout}

1) Functional site space layout: The function space layout of site is built in view of different site landscape plaques, based on establishment of site overall layout, under guidance of overall planning and project concept. It includes two aspects as follows: firstly, ensure the layout, flow lines, levels should be uniform in the overall space layout; secondly, according to the different characteristics of relics plaques, distinguish different function divisions which are organized reasonably in sequence, make the site reflect the cultural atmosphere based on following the original layout of site.

2) Landscape space sequence organization: Landscape space sequence organization is the important content of space planning which make the site and landscape perfect through reasonably organizing the site division and space levels. The organization method of landscape space sequence has been studied in this paper through planning of road system and sight line system.

\section{a) Road system planning}

Features: The road system of relics park has a unique function and features in addition to guiding function with respect to ordinary park. Firstly, the roads in relics park have historic features, that is, namely it can provide related historical information of the site. However most of the road entities have been disappeared, so it is important to recovery the roads; secondly, the relics roads also have cultural features, because the interior space of the site is the space connected by roads link, it mainly expresses its cultural characteristics through road pavement and greening etc.

Type: According to function, the roads of relics park are divided into two types of road traffic and sightseeing.

The traffic roads mainly undertake the functions of traffic dispersion and experience, compared with ordinary parks, of which the layout and streamline aim to meet relics and landscape display and experience functions. Especially in the protection areas, the road should be strictly designed in accordance with the original layout to play diversion, guidance and implied roles.

The sightseeing roads have taken the function to connect interior functional zones of the site park, with flexible type, are the primary means of accomplishing relics landscape sequence. Furthermore the sightseeing roads also have the function of displaying and experience landscape. 


\section{b) Line of sight planning}

The sight organization in relics park can not only strengthen cultural landscape features, but also meet various functions of the park, make the whole park uniform in landscape.

There are two primary methods used in line of sight planning organization in relics park as follows: dominant corridor sight planning and hidden corridor sight planning. Dominant corridors are mainly aimed to guide the theme landscape sight, directly show the theme landscape within scope of the sight through several main axis, which focus on permeability between scenic spots, and complete line of sight and streamline planning through organization of various scenic spots arrangement in axis. However hidden corridors are designed in view of weak cultural feature of some scenic spots, through a series of line of sight guidance to show its attractions rather than showing in the sight directly.

\section{Site Landscape Display Space Planning}

The Site landscape display space planning refers to the planning and design for the building facilities, public service facilities and green landscape which are used to display relics, such as site museum, relics digging display area, which should be designed based on strict site protection with main function to protect the site itself and its environment, and rich styles and space levels of relics display through various measures.

\section{CONSTRUCTION OF LANDSCAPE SPACE CREATING SYSTEM IN RELICS PARK}

Through respectively researching on the creating methods of three levels of overall, medium and tiny of cultural landscape space and physical landscape space, the characteristics of the creating system are summarized as follows:

Overall level: The cultural landscape has emphasis on cultural characteristics of the whole park, through abstracting the cultural theme, and then put forward the concept of planning; while the entity landscape has emphasis on structure integration and streamlines arrangement of the overall layout.

Medium level: The cultural landscape is refined and summarized in view of the site culture plaques with division concept of project planning; while the entity landscape takes arrangement and sequence organization of functional landscape space structure through traffic and sight planning.

Tiny level: The cultural landscape mainly concludes the intention expression of the site through refinement of site culture elements; while the entity landscape space is planned in view of relics itself and its carriers, including aesthetic treatment and greening design of relics landscape.

\section{CONCLUSIONS}

Through researching on theory of landscape space of relics park, the significance of space construction in relics park planning and the necessity of improving construction measures have been proposed by this paper, what is more a space construction theory of relics park planning has been put forward. In researching on construction methods of cultural space and entity space in relics space construction, an overall framework finally has been proposed from three levels of overall, medium and tiny levels which have different contents in different ranges.

\section{REFERENCES}

[1] Zhang Song. Introduction to the historical city protection - a holistic approach to cultural heritage and historical environmental protection[M] China Construction Industry Press, 2001.

[2] Ruan Yisan, Wang Jinghui, Wang Lin. Theory and planning on Prevention and Protection of Historical and Cultural Cities [M]. Tongji University Press, 2002. 